\title{
Cross-cultural differences in the perception of facial expressions of ambiguous Noh faces
}

\author{
NAOYUKI OSAKA \\ Otemon-Gakuin University, Ibaraki, Osaka, Japan
}

\begin{abstract}
A multidimensional scaling technique was applied in the judgment of ambiguous facial expressions using 10 Japanese Noh faces. Subject groups from West Germany and Japan rated 45 pairs of faces using a 9-point similarity scale. A derived stimulus configuration revealed cross-cultural differences in the perception of ambiguous faces.
\end{abstract}

Researchers since Darwin (1872) have used the rating method to investigate the interpretation of facial expression. In a typical experimental condition, the subject is asked to rate or interpret which emotion a given face stimulus expresses. Schlosberg (1952) found that the confusion, for example, between anger and fear, was due to physical similarity of the facial expressions associated with different emotions.

The stimuli employed in face perception or face recognition experiments have usually been photographs of actors/actresses portraying various emotions or composite faces made from Identi-Kit or Photofit (Davies, Ellis, \& Shepherd, 1981). By using such photographs or composite faces, researchers can control cue saliency in faces (Shepherd, Davies, \& Ellis, 1981) involving featural information, contour cues provided by the shape of the face, and the relative distance between features and contours. Although these stimuli may be salient faces that express some emotion, these two techniques for constructing facial images do not always result in good representations of facial expression (Yount \& Laughery, 1982). To determine whether there were cross-cultural differences in identifying and recognizing emotion in faces, in this experiment, I used Japanese Noh faces, which are artificial, sometimes ambiguous, faces that may be interpreted as expressing varied emotion (e.g., one face may be interpreted as either happy or sorrowful) (Osaka, 1984).

\section{METHOD}

\section{Stimuli}

Ten Noh faces, as shown in Figure 1, were employed. In Japanese Noh play tradition, the faces are called (1) Okina, (2) Otobide, (3) Obeshimi, (4) Kaminari, (5) Koomote, (6) Magojiro, (7) Komachiroujyo, (8) Douji, (9) Reionna, and (10) Hannya. Faces 3, 4, and 10 are salient in their expressions of anger, whereas Faces 1 and 8 express happiness, and Face 2 appears surprised. The most interesting faces are

This work was supported by grants from DAAD (West Germany) and JSPS (Japan). Part of the data were collected during the author's stay at the Institute for Medical Psychology, University of Munich, 1985. Thanks are due to David Pöppel and Brigitte Jordan for their help in collecting the data and to Ernst Pöppel for his advice during the experiment. The author's mailing address is: Department of Psychology, Otemon-Gakuin University, Ibaraki, Osaka 567, Japan.
5,6 , and 7 , because their expressions are essentially ambiguous. The purpose of the present research was to investigate cross-cultural differences in the perception of expressions of these ambiguous Noh faces.

\section{Procedure}

Forty-five pairs were made from all possible combinations of these 10 faces, and the subject was asked to rate each pair's similarity using a 9-point similarity scale (1 for highly dissimilar and 9 for highly similar). Each stimulus was presented on a screen, using a slide projector. The order of presentation was randomized, and each pair was presented for $10 \mathrm{sec}$ with 10-sec intertrial intervals. Although perception of presented unfamiliar faces is significantly impaired by brief presentations, this inspection time was long enough for facial expressions to be rated. Each subject made two ratings of each stimulus pair. The stimulus presentation sequence was controlled automatically by a logic module incorporating counter.

\section{Subjects}

Twenty scientists (10 males and 10 females) from West Germany (their ages ranged from 21 to 43 years) and 20 college students ( 10 males and 10 females) from Japan (ages ranged from 20 to 24 years) served as subjects. All subjects had normal or corrected-to-normal visual acuity.

\section{Multidimensional Scaling}

Each of $4010 \times 10$ input triangle matrices contained measures of a subject's judgments of similarity of the corresponding stimulus pairs. Each matrix was processed using a weighted individual-difference MDS program (INDSCAL; Takane, Young, \& de Leeuw, 1977). Two dimensions were derived using the weighted Euclidean model (Young \& Lewyckyj, 1979), since previous analyses of these data had indicated that the two-dimensional solution was most interpretable (Abelson \& Sermat, 1962).

\section{RESULTS AND DISCUSSION}

The overall stress and RMS (root-mean-square) values were found to be 0.204 and 0.78 , respectively, which were moderately good. The average RMS values for subjects (for each matrix) varied from 0.45 to 0.94 . Individual weight space was measured, and averaged stimulus configuration was generated. Figures 2 and 3 show, in terms of a spatial mapping, the results for the Japanese and German subjects, respectively, on two dimensions of expressed emotion (Schlosberg, 1952): (1) pleasantnessunpleasantness and (2) tenseness-sleepiness. Numbers that are placed higher on Dimension 2 represent pictures judged to express more tension; numbers placed farther to the right on Dimension 1 represent pictures judged to express greater unpleasantness. As is obvious in the 

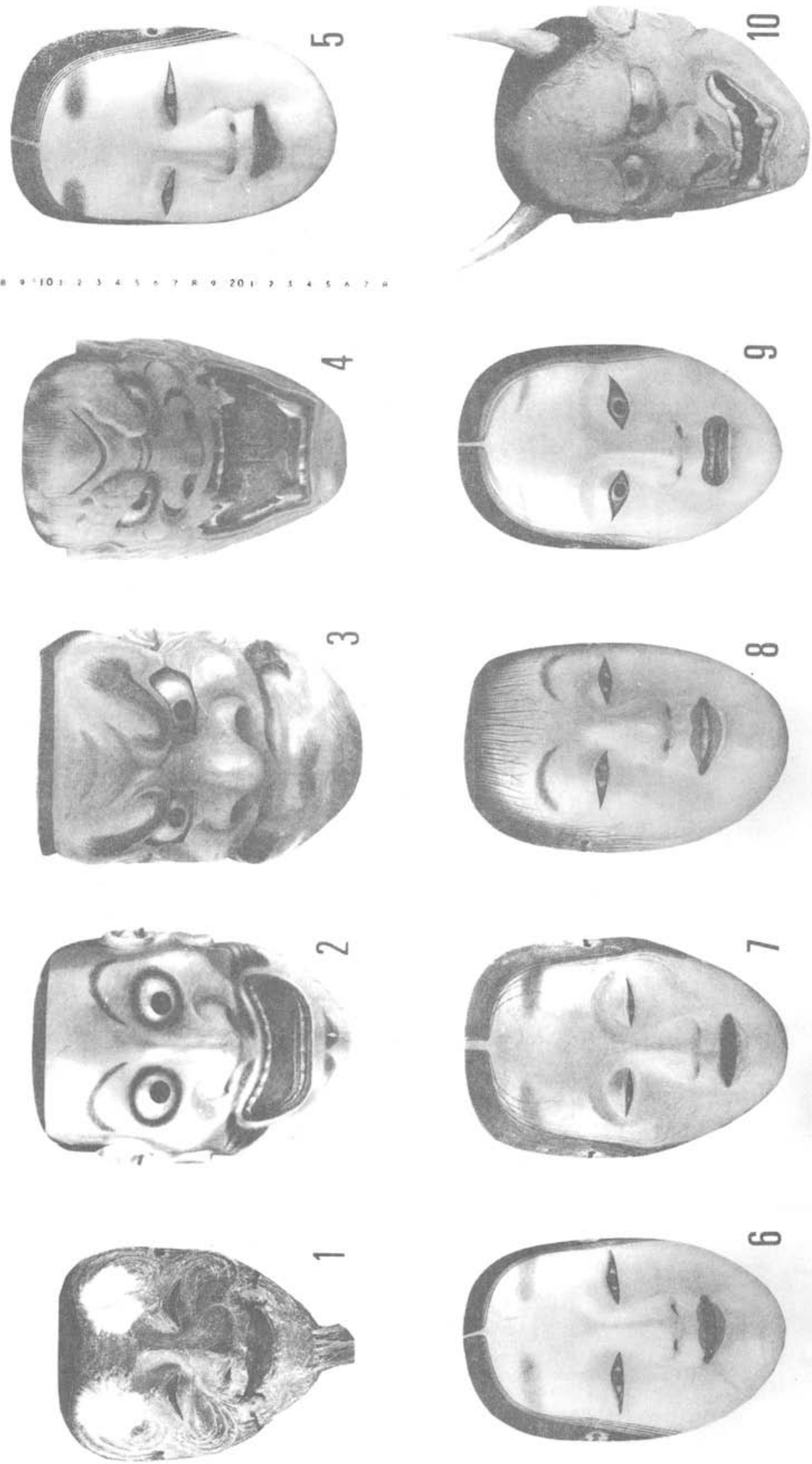

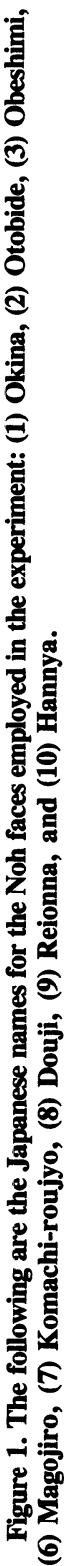




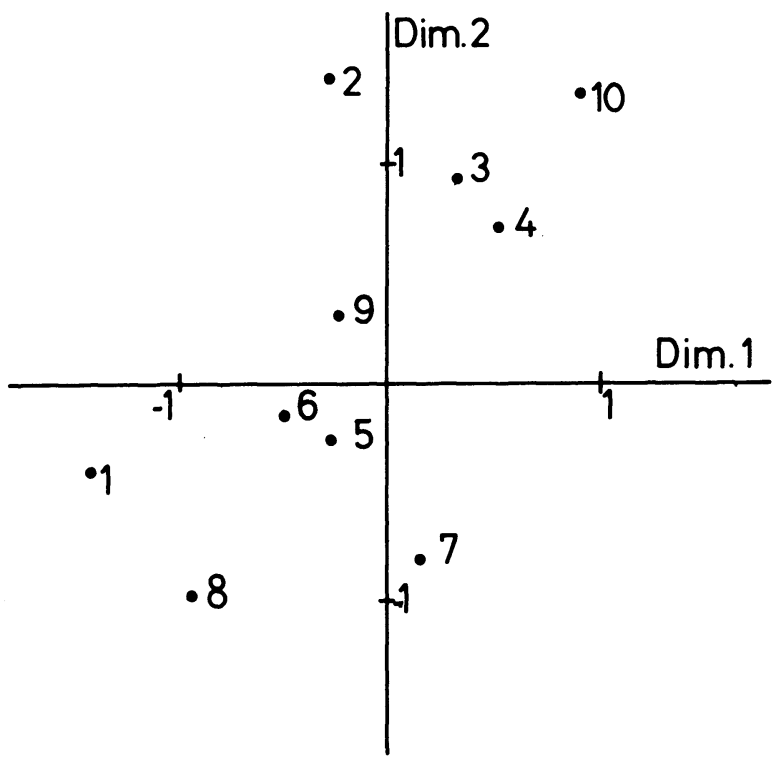

Figure 2. The derived stimulus configuration for subjects from Japan. The numbers correspond to the numbered stimulus faces shown in Figure 1. Dimension 1 represents pleasantnessunpleasantness judgments of expression; Dimension 2 represents tenseness-sleepiness judgments of expression. Numbers that are higher on Dimension 2 represent faces judged to express more tension; numbers placed farther to the right on Dimension 1 represent faces judged to express greater unpleasantness.

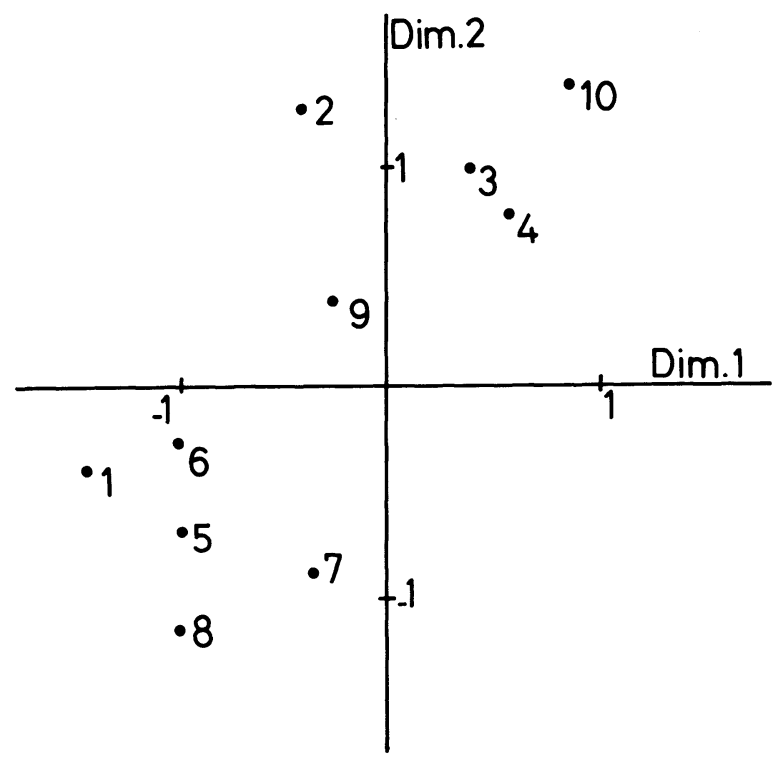

Figure 3. The derived stimulus configuration for subjects from Germany. The numbers correspond to the numbered stimulus faces shown in Figure 1. Dimension 1 represents pleasantnessunpleasantness judgments of expression; Dimension 2 represents tenseness-sleepiness judgments of expression. Numbers that are higher on Dimension 2 represent faces judged to express more tension; numbers placed farther to the right on Dimension 1 represent faces judged to express greater unpleasantness. figures, the two groups of subjects rated nonambiguous faces similarly on both dimensions. In terms of crosscultural differences, it appears that the German subjects judged the ambiguous faces $(5,6$, and 7$)$ as expressing more pleasant emotion (i.e., smiling) than did the Japanese subjects. The German subjects' ratings of ambiguous faces tended to cluster along Dimension 1, suggesting that the German subjects seemed to accept them as less ambiguous than Japanese subjects did. It is likely that Japanese and German subjects would use different facial features to describe and recognize the ambiguous Noh faces.

This finding can be interpreted as demonstrating a different culturally based perception of Noh faces for both groups, even though it is inherently more difficult for German subjects to recognize oriental-culture faces, such as Noh faces, than white faces. By applying the multidimensional scaling technique, Shepherd and Deregowski (1981) found that African and European groups of subjects used different attributes for judging two sets of cross-racial faces. Although superiority in recognizing faces of one's own race has been reported in a number of studies (Ellis, Davies, \& McMurran, 1979; Goldstein \& Chance, 1978; Goldstein, Johnson, \& Chance, 1979), Elliott, Wills, and Goldstein (1973) found that training with oriental faces (learning their facial schema) did improve performance in the recognition of oriental faces. The differences found between the German and Japanese subjects in their responses to ambiguous Noh faces can be explained in terms of schema-dependent cross-cultural differences.

\section{REFERENCES}

Abelson, R. P., \& Sermat, V. (1962). Multidimensional scaling of facial expressions. Journal of Experimental Psychology, 63, 546-554.

DARWIN, C. (1872). The expression of the emotions in man and animals. London: Murray.

Davies, G., Ellis, H., \& ShePherd, J. (1981). Perceiving and remembering faces. London: Academic Press.

Elliott, E. S., Wills, E. J., \& Goldstein, A. G. (1973). The effects of discrimination training on the recognition of white and oriental faces. Bulletin of the Psychonomic Society, 2, 71-73.

Ellis, H. D., Davies, G. M., \& McMurran, M. M. (1979). Recall of white and black faces by white and black witnesses using the Photofit system. Human Factors, 21, 55-59.

Goldstein, A. G., \& Chance, J. (1978). Judging face similarity in own and other races. Journal of Psychology, 98, 185-193.

Goldstein, A. G., Johnson, K. S., \& Chance, J. E. (1979). Does fluency of face description imply superior face recognition? Bulletin of the Psychonomic Society, 13, 15-18.

OsaKa, N. (1984, September). Analysis of individual weight space differences in percept and imaginary map using INDSCAL model. Paper presented at the 49th annual meeting of the Japanese Psychological Association.

SCHLOSBERG, H. (1952). The description of facial expressions in terms of two dimensions. Journal of Experimental Psychology, 44, 229-237.

Shepherd, J. W., Davies, G. M., \& Ellis, H. D. (1981). Studies of cue saliency. In G. Davies, H. Ellis, \& J. Shepherd (Eds.), Perceiving and remembering faces. London: Academic Press.

ShePherd, J. W., \& DeREgowsKI, J. B. (1981). Races and faces: A comparison of the responses of African and Europeans to faces of the same and different races. British Journal of Social Psychology, 20, 125-133. 
Takane, Y., Young, F. W., \& De Leeuw, J. (1977). Nonmetric individual differences multidimensional scaling: An alternating least squares method with optimal scaling features. Psychometrika, 42, 7-67. Young, F. W., \& LeWYCKYJ, R. (1979). ALSCAL-4 user's guide. Chapel Hill, NC: L. L. Thurstone Psychometric Lab.
Yount, M. B., \& LAUGHeRY, K. R. (1982). Facial memory: Constructing familiar and unfamiliar faces. Bulletin of the Psychonomic Society, 19, 80-82.

(Manuscript received for publication July 11, 1986.) 